\title{
Variation in patients' perceptions of elective percutaneous coronary intervention in stable coronary artery disease: cross sectional study
}

\author{
Faraz Kureshi NIH T-32 cardiovascular outcomes research fellow ${ }^{12}$, Philip G Jones senior \\ statistician $^{1}$, Donna M Buchanan cardiovascular outcomes researcher/manager ${ }^{12}$, Mouin S Abdallah \\ cardiology fellow ${ }^{12}$, John A Spertus Lauer/Missouri endowed chair and tenured professor of \\ medicine $^{12}$
}

${ }^{1}$ Saint Luke's Mid America Heart Institute, Kansas City, MO 64111, USA; ${ }^{2}$ University of Missouri- Kansas City, Kansas City, MO, USA

\begin{abstract}
Objectives To assess the perceptions of patients with stable coronary artery disease of the urgency and benefits of elective percutaneous coronary intervention and to examine how they vary across centers and by providers.
\end{abstract}

Design Cross sectional study.

Setting 10 US academic and community hospitals performing percutaneous coronary interventions between 2009 and 2011.

Participants 991 patients with stable coronary artery disease undergoing elective percutaneous coronary intervention.

Main outcome measures Patients' perceptions of the urgency and benefits of percutaneous coronary intervention, assessed by interview. Multilevel hierarchical logistic regression models examined the variation in patients' understanding across centers and operators after adjusting for patient characteristics, using median odds ratios.

Results The most common reported benefits from percutaneous coronary intervention were to extend life ( $90 \%, \mathrm{n}=892$; site range $80-97 \%)$ and to prevent future heart attacks ( $88 \%, n=872$; site range $79-97 \%)$. Although nearly two thirds of patients $(n=661)$ reported improvement of symptoms as a benefit of percutaneous coronary intervention (site range $52-87 \%)$, only $1 \%(n=9)$ identified this as the only benefit. Substantial variability was noted in the ways informed consent was obtained at each site. After adjusting for patient and operator characteristics, the median odds ratios showed significant variation in patients' perceptions of percutaneous coronary intervention across sites (range 1.4-3.1) but not across operators within a site.

Conclusion Patients have a poor understanding of the benefits of elective percutaneous coronary intervention, with significant variation across sites. No sites had a high proportion of patients accurately understanding the benefits. Coupled with the wide variability in the ways in which hospitals obtain informed consent, these findings suggest that hospital level interventions into the structure and processes of obtaining informed consent for percutaneous coronary intervention might improve patient comprehension and understanding.

\section{Introduction}

Although elective percutaneous coronary intervention can alleviate the symptoms of angina in patients with stable coronary artery disease, numerous studies and meta-analyses have shown that it does not prevent myocardial infarction or death, as compared with optimal medical treatment alone..$^{1-5}$ Accordingly, the decision to proceed with percutaneous coronary intervention should involve a discussion with patients as to whether or not the benefits of improvement in symptoms outweigh the risks and costs (for example, the co-pay for the procedure itself and the long term costs of additional medications, such as thienopyridines), as compared with medical treatments alone. Patients' with a better understanding about treatments may be more involved in shared decision making, thus being more likely to receive care that is aligned with their goals and ensuring that practice variations in treatment for stable coronary artery disease result from patients' rather than from physicians' preferences. ${ }^{6-11}$ For patients to meaningfully participate in decision making about treatment for their stable coronary artery disease, a sound understanding of the benefits, risks, and alternatives of elective percutaneous coronary intervention is essential.

Substantial evidence indicates that patients have a limited understanding of the benefits of elective percutaneous coronary intervention in stable coronary artery disease and believe that 
it is performed primarily to prevent heart attacks and improve survival. ${ }^{12-19}$ While work has explored associations between patient factors and patient perceptions of percutaneous coronary intervention, ${ }^{12} 141519$ no study has examined whether patients' perceptions vary by the hospital where they are treated or as a function of the interventional cardiologist performing the procedure. Investigating sources of patient variation is extremely important in determining whether interventions and efforts to better educate patients should extend beyond targeting patient level factors alone, such as education, literacy, and numeracy.

To tackle this gap in knowledge, we conducted a multicenter investigation of the understanding of patients with stable coronary artery disease of the benefits of elective percutaneous coronary intervention and examined if patients' perceptions varied across centers, across operators within a center, or both. By examining if variation exists across these levels, we sought to identify whether operator level or hospital level factors influence patients' perceptions and to illuminate potential targets for quality improvement efforts to improve patients' understanding.

\section{Methods}

\section{Study design, setting, and participants}

This cross sectional study was an extension of the Personalized Risk Information Services Manager (PRISM) study (NCT01383382), a nine center study of academic medical centers and large community hospitals across the United States, that sought to implement individualized model estimates for risk of bleeding in each patient undergoing percutaneous coronary intervention. ${ }^{20}$ An additional site, which prospectively collected the same information about patients' knowledge of the benefits of percutaneous coronary intervention, was also included. During September 2009-2011, we invited consecutive patients undergoing an elective percutaneous coronary intervention $(n=3299)$ across 10 centers (see supplementary appendix table 1) from nine states to participate in an interview assessing their understanding of the urgency and benefits of their procedure. Trained research coordinators conducted structured interviews with patients once they were clinically stable, usually between four and six hours after the percutaneous coronary intervention, when the interventional cardiologist was not present. To focus on patients for whom the primary indication for intervention was symptomatic improvement, we specifically excluded those with acute coronary syndromes (ST segment elevation myocardial infarction, non-ST segment elevation myocardial infarction, unstable angina) who had urgent or emergent procedures ( $\mathrm{n}=2295)$. Accordingly, we restricted our analytic cohort to those undergoing an elective percutaneous coronary intervention with a diagnosis of stable coronary artery disease $(n=1004)$.

\section{Describing the informed consent process}

During the PRISM implementation process, the study's central implementation team collected a detailed analysis of the process of obtaining informed consent (for example, the location where consent was signed, who obtained it, whether supplemental educational documents were used). This was supplemented with a detailed, qualitative assessment by each of the sites' principal investigators of the process by which informed consent was obtained at each institution.

\section{Measurement of patient perceptions and clinical data}

The primary measurement for our analysis was based on the answers from two previously used questions (see supplementary appendix figure 1) that had been designed to assess the patients' understanding of the urgency and benefits of their procedure. ${ }^{17}$ The first question dealt with whether the patients perceived the procedure to be elective or emergent, or if they were unsure. The second was a list of potential benefits from the procedure (extend life, prevent myocardial infarction, save life, decrease symptoms, improve stress test abnormality, and other, which permitted a narrative description), from which they could select as many as they perceived to be a benefit of percutaneous coronary intervention.

During the interview we collected information on demographic and clinical characteristics, including sex, race, marital status, smoking status, medical history (prior myocardial infarction, percutaneous coronary intervention, and coronary artery bypass grafting), and socioeconomic status, and information based on the disease specific Seattle angina questionnaire and the Rose dyspnea scale to assess health status. The Seattle angina questionnaire is a validated, disease specific health status measure that quantifies five dimensions of health in patients with coronary artery disease: physical limitations, stability of angina, frequency of angina, satisfaction with treatment, and quality of life. Possible scores range from 0-100, with lower scores noting worse effects related to angina. ${ }^{21}$ The Rose dyspnea scale is a questionnaire that assesses a patient's level of dyspnea while participating in common activities, with scores ranging from 0-4 (higher scores indicating worse dyspnea). ${ }^{22}$ This scale has been shown to be sensitive to change in patients with coronary artery disease. ${ }^{23}{ }^{24}$

We obtained the characteristics of the interventional cardiologists (operators) through national provider identifier numbers, state licensing websites, the certification verification website of the American Board of Internal Medicine, practice websites, the US National Library of Medicine and National Institutes of Health website, or direct communication with the site. Information was obtained on age, sex, medical school, years in practice (since graduation from last completed graduate medical education training program), board certification status, and number of scientific publications.

\section{Statistical analysis}

To examine variation in the perceptions of the benefits from percutaneous coronary intervention in patients with stable coronary artery disease, we first calculated the rates of each perceived benefit from the questionnaire for the overall study population and then stratified by site, using descriptive analyses. We then created three level (patient, operator, and hospital), multivariable hierarchical models to assess the relation of each outcome (patient perceived sense of urgency or benefit from percutaneous coronary intervention) with patient level and operator level factors. A multilevel analysis allows for the incorporation of variables measured at different levels of the hierarchy (patient, operator, and hospital), where the perceptions of patients under the care of one operator, or within one site, may be correlated. This avoids overestimating the significance of statistical associations. ${ }^{25}$ To examine the variation across providers and hospitals, after adjusting for patient level and physician level characteristics, we calculated the median odds ratios by treating patient and operator factors as fixed effects (allowing for adjustment of median odds ratios), while site and operator within a site were treated as random effects. We 
simultaneously adjusted operator and hospital median odds ratios to estimate the independent effect of each. The median odds ratio can be interpreted as the variability with which two statistically similar patients with stable coronary artery disease would hold a certain perception of percutaneous coronary intervention if they were treated by a different operator or at a different site. A median odds ratio value is always 1 or greater and if the confidence interval does not include 1, then it supports the conclusion that significant variation exists across that unit of analysis (physician or hospital). ${ }^{26} 27$

All analyses were conducted using SAS statistical software, version 9.3 (SAS Institute), with a two sided significance level being defined as $\mathrm{P} \leq 0.05$ or $95 \%$ confidence intervals excluding unity.

\section{Results}

Among 1004 patients with stable coronary artery disease having an elective percutaneous coronary intervention across 10 sites, 991 patients (98.7\%) participated in the interview and answered questions to assess their perceptions of the urgency and benefits of percutaneous coronary intervention done by 135 operators across 10 sites. Most interventional cardiologists $(89 \%, \mathrm{n}=120)$ each performed percutaneous coronary intervention on 1-14 patients with stable coronary artery disease, whereas $15(11 \%)$ did so on more than 15 patients each (table $1 \Downarrow$ ). Supplementary appendix table 2 describes the number of patients and operators at each site.

The process of obtaining informed consent differed considerably across institutions (table $2 \Downarrow$ ). At some sites the operators or fellows were the ones who oversaw the consent form being signed, whereas at others it was predominantly the nurses. Although consent in most sites was obtained in the hospital, one site also obtained consent prior to the day of the procedure (rarely). One site consistently provided educational materials to patients undergoing elective percutaneous coronary intervention, whereas others did so occasionally, rarely, or never.

\section{Patient and operator characteristics}

Table $3 \Downarrow$ describes the patient population, which had a mean age of 65 (SD 10.6) years, was predominantly male $(75.2 \%$, $\mathrm{n}=745)$ and of white race $(92.9 \%, \mathrm{n}=906)$, and had at least a high school education $(92.5 \%, \mathrm{n}=917)$. Only six $(0.6 \%)$ of the patients had no form of medical insurance. Approximately $44 \%$ $(n=432)$ of the patients reported having had a previous percutaneous coronary intervention procedure and $85 \%(n=842)$ had symptoms of chest pain or shortness of breath on the basis of scores on the Seattle angina questionnaire and Rose dyspnea scale prior to their procedure.

Table $4 \Downarrow$ describes the characteristics of the 135 interventional cardiologists performing the procedures. The mean age and years of practice were 50.7 (SD 8.4) and 17.6 (9.3) years, respectively. Most of the cardiologists were graduates of US or Canadian medical schools $(76 \%, \mathrm{n}=103)$ and certified by the American Board of Internal Medicine in cardiovascular diseases $(96 \%, \mathrm{n}=130)$ and interventional cardiology $(82 \%, \mathrm{n}=111)$. Only eight $(6 \%)$ were women.

\section{Perceptions of the urgency and benefits of percutaneous coronary intervention}

Twenty per cent (site range 4-38\%) of patients classified their procedure as "emergent," in spite of the fact that all were elective. Table $5 \Downarrow$ describes the average and variability across sites in patients' identified benefits of percutaneous coronary intervention (also see fig $1 \Downarrow$ ). Although patients often reported multiple benefits from the procedure (table $6 \Downarrow$ ), those most commonly reported were to extend life (90\%; site range $80-97 \%$ ) and to prevent future heart attacks $(88 \% ; 79-97 \%)$, followed by to save life $(69 \% ; 31-85 \%)$. Although $67 \%$ (site range $52-87 \%$ ) of patients did report that percutaneous coronary intervention was done for improvement of symptoms, only $1.0 \%$ identified this as the only benefit from treatment.

\section{Association of patient and operator characteristics with perceptions of benefits}

In general, few patient characteristics were associated with specific perceived benefits. Supplementary appendix table 3 describes the odds ratio of each patient and operator characteristics with specific patient perceptions or benefits from percutaneous coronary intervention. The strongest patient level predictor that was associated with the belief that the procedure would improve symptoms was angina (odds ratio $0.75,95 \%$ confidence interval 0.71 to 0.78 , for each five point greater angina frequency score, where higher scores indicate less angina). Younger patients were more likely to believe that percutaneous coronary intervention would prevent future myocardial infarctions, save their life, and decrease their symptoms. Married patients thought the procedure would prevent myocardial infarction, whereas less educated patients thought it was emergent (even though all cases were elective) and more educated patients thought that it would improve a stress test abnormality. Age was the only operator characteristic independently associated with patients' perceived benefit, with patients who had their procedures done by older interventional cardiologists believing that the percutaneous coronary intervention would extend their lives.

\section{Variation in patient perceptions across centers and operators within hospitals}

After adjusting for the characteristics of patients and operators (tables 1 and 2), significant variation in patients' perceptions of percutaneous coronary intervention was observed across hospitals, as the median odds ratios excluded 1.00 (fig $2 \Downarrow$ ). For example, the median odds ratio of the patients' perception that percutaneous coronary intervention would prevent a future myocardial infarction across centers was 1.72 (95\% confidence interval 1.29 to 2.94), meaning that for two patients with similar observed covariates who have percutaneous coronary intervention at two randomly selected hospitals, one patient would have a $72 \%$ greater odds than the other of believing that the procedure would prevent a future myocardial infarction. In contrast, there was no significant variation in patients' understandings of the benefits of percutaneous coronary intervention across physicians within a hospital, as all of the median odds ratios included 1.00. For the patients' perceptions that percutaneous coronary intervention would decrease their symptoms, the median odds ratio was 1.74 (1.36 to 2.84 ) across hospitals and 1.00 (1.00 to 1.40$)$ among operators within a hospital.

\section{Discussion}

Because patients generally prefer to be informed and involved in deciding on their treatments, even if they are comfortable relinquishing the technical details of treatment to their physicians, it is critically important that they understand the benefits of treatment to them. ${ }^{28-31}$ From this multicenter study we found that the overwhelming majority of patients with stable coronary artery disease undergoing an elective percutaneous 
coronary intervention overestimate the benefits of the procedure and have a poor understanding that its primary indication is for improvement of symptoms. Specifically, patients predominantly believed that the procedure would extend their life and prevent a heart attack, despite abundant clinical evidence to the contrary. ${ }^{134}$ While almost two thirds of patients noted symptom relief as a benefit of the procedure, only $1.0 \%$ identified this as the only benefit of percutaneous coronary intervention. These findings highlight persistent problems in patients' understandings about the benefits of elective percutaneous coronary intervention in the management of stable coronary artery disease.

Moreover, we documented substantial variation in the process of obtaining signed informed consent across the 10 centers, and that after adjustment for patient and operator characteristics, patients' perceptions of the benefits of percutaneous coronary intervention varied significantly across institutions, but not across operators within an institution. Collectively, these findings suggest that important opportunities exist to improve the process, reliability, and quality of informed consent for percutaneous coronary intervention and hospital level interventions may be an important step.

\section{Strengths and limitations of this study}

The primary strengths of our study include being the largest investigation of patient perceptions of percutaneous coronary intervention in the management of stable coronary artery disease, its contemporary nature, and the use of multiple sites, varying from large, academic centers to private hospitals. Moreover, by including multiple sites and a novel approach, we have described substantial variability by which informed consent is obtained at each site and demonstrated significant variation of patients' perceptions across sites.

Our results should be interpreted in the context of several potential limitations. Firstly, we did not directly observe or obtain detailed information on the informed consent process or discussions in the clinic when the decision was made to proceed with percutaneous coronary intervention. This precluded us from knowing whether there were differences in the time spent or techniques used to inform patients of the benefits of percutaneous coronary intervention. Observational research with direct audio or video recording of these discussions would have potentially provided additional insights into the variability in educating patients about the benefits of percutaneous coronary intervention. Secondly, we did not assess operators' or referring physicians' perceptions of the benefits of percutaneous coronary intervention in this cohort with stable coronary artery disease as it is certainly possible that the operators or referring physicians themselves had misperceptions that they used to inform patients. ${ }^{12} 32$ Thirdly, we only surveyed patients who chose to have an elective percutaneous coronary intervention. Patients who elected not to have the procedure may have had a more accurate understanding of its benefits. However, this does not alter our observation that most of the patients with stable coronary artery disease who were having an elective percutaneous coronary intervention overestimated its benefits. Fourthly, although interviews and data collection were to be done in a standardized manner based on protocols, it is not possible to be sure that the variation across sites might not be attributable to differences in the techniques of the data coordinators in interviewing patients. Similarly, there are no validated instruments to assess patients' understanding of the benefits of percutaneous coronary intervention and we relied on an instrument that we had used previously ${ }^{17}$ and that seemed to be accurately understood by patients. Finally, given the observational nature of data, we cannot exclude unmeasured confounding.

\section{Comparison with other studies}

Our findings are consistent with, and considerably extend, previously published studies that have examined the perceptions of patients with stable coronary artery disease about percutaneous coronary intervention. ${ }^{12-19}$ The patients' perceptions of improved survival (90\%) and prevention of a myocardial infarction (88\%) from our study are even larger than those in a study from greater than a decade ago, which reported that $71 \%$ of patients believed that percutaneous coronary intervention would extend their life and $75 \%$ reported that it would prevent a heart attack. ${ }^{14}$ Similarly, a multicenter study found that $83 \%$ of 309 surveyed patients with stable coronary artery disease who were offered revascularization procedures after an elective coronary angiography thought that percutaneous coronary intervention would improve their survival. ${ }^{19} \mathrm{~A}$ more contemporary study found that $82 \%$ and $88 \%$ of 153 patients with stable coronary artery disease undergoing elective coronary angiography at one site believed that percutaneous coronary intervention would decrease their risk of fatal and future myocardial infarction, respectively..$^{12}$ However, limitations in the generalizability of these and previous studies are small cohort sizes, single center studies, ${ }^{16}$ or publication prior to the highly publicized COURAGE trial. ${ }^{233-35}$ Our study expands on this pioneering work by providing a more generalizable insight into the perceptions of patients with stable coronary artery disease of the benefits of percutaneous coronary intervention in modern day practice by including nearly 1000 patients across 10 sites and explicitly examining if these patients' perceptions varied across hospitals and physicians.

\section{Possible explanations and implications for clinicians, policy makers, and researchers}

Unwarranted variations in healthcare delivery for preference sensitive conditions, such as percutaneous coronary intervention in stable coronary artery disease, are of extreme interest as observed differences are not attributable to patients' values and preferences or the severity of their underlying illness. ${ }^{36}$ Interventions to better educate patients so they are able to express an informed preference for treatment while taking into account the timing, structure, and incentives of the decision making process have been proposed as ways to deliver patient centered care. ${ }^{37}$ For patients with stable coronary artery disease undergoing an elective percutaneous coronary intervention, the informed consent process provides a vital opportunity to review the benefits, risks, and alternatives of percutaneous coronary intervention with patients. Congruent with our findings of significant variability in patients' understanding of the benefits of the procedure across hospitals, we found considerable variation in how informed consent was obtained at each site. While some sites had lower frequencies of patient misperceptions of the benefits and purpose of their elective percutaneous coronary intervention, no site had a majority of patients with stable coronary artery disease appreciating that the primary benefit of elective percutaneous coronary intervention was the relief of symptoms. Future studies should examine interventions that standardize the consent process and examine the incremental benefits of additional education materials, videos, decision aids, formal shared medical decision making tools, or multidisciplinary teams, such as Heart teams, to improve knowledge transfer. ${ }^{38-40} 204142$ Our results suggest that there is great opportunity for the routine use of such tools 
to improve the informed consent process for percutaneous coronary intervention.

While our study was unable to explore hospital level factors driving site level variation because of the limited number of sites, it is possible that organizational culture contributes to both the different processes of obtaining informed consent and the amount of information conveyed. The impact of organizational culture on performance and outcomes has been well recognized in other disciplines ${ }^{43}{ }^{44}$; however, there has been the development of emerging interest in the healthcare discipline. ${ }^{43-46}$ Although there is no consensus on the definition and measurement of organizational culture, some agreed upon tenets are that it does exist, is unique to each institution, and is a product of groups, not individuals. ${ }^{47}{ }^{48}$ While strong evidence linking an association between organizational culture and quality of healthcare is limited because of too few studies, there are examples of top performing healthcare institutions in various domains (for example, patient safety, quality of care for acute myocardial infarction) the success of which is primarily attributed to constructing a culture promoting quality. ${ }^{43}{ }^{44}$ Prioritizing the importance of successfully communicating the benefits, risks, and alternatives of elective percutaneous coronary intervention to patients with stable angina, as a foundation for shared medical decision making, would likely be accelerated by creating an organizational culture that emphasized the importance of patient centered care, provided a supporting infrastructure of educational materials, and created a reproducible process that was performed in each and every patient.

\section{Conclusions}

We interviewed a large cohort of patients with stable coronary artery disease undergoing an elective percutaneous coronary intervention across multiple US hospitals and found that patients substantially overestimate the benefits of the procedure. Most importantly, we found that after adjusting for patient and operator characteristics, there was substantial variation in patients' perceptions across sites, but not across operators within a site. Coupled with the wide variability in the ways in which hospitals obtain informed consent, these findings suggest that hospital level interventions into the structure and processes of obtaining informed consent for percutaneous coronary intervention might improve patient understanding.

Contributors: All coauthors authors have fulfilled authorship criteria per ICMJE guidelines and have approved the manuscript and this submission. All coauthors provided substantial contributions to the conception or design of the work, or acquisition, analysis, or interpretation of data for the work, and helped with drafting the work or revising it critically for important intellectual content. All coauthors approve this version of the manuscript and agree to be accountable for all aspects of the work in ensuring that questions related to the accuracy or integrity of any part of the work are appropriately investigated and resolved. FK, PGJ, and JAS are guarantors for the study, had full access to all of the data in the study, and take responsibility for the integrity of the data and the accuracy of the data analysis. The funding agencies had no role in data collection, analysis, interpretation, or the decision to submit the results.

Funding: The study was supported by an American Heart Association Outcomes Research Center grant (0875149N) and the National Heart Lung and Blood Institute (R01-HL096624). While the grants supported different aims, they employed the same patient population, study design, and data collection, and were combined for the purpose of these analyses. FK received support from National Institutes of Health grant T32HL110837. The funding agencies had no role in data collection, analysis, interpretation or the decision to submit the results.
Competing interests: All authors have completed the ICMJE uniform disclosure form at www.icmje.org/coi_disclosure.pdf (available on request from the corresponding author) and declare: the study was supported by an American Heart Association Outcomes Research Center grant (0875149N) and the National Heart Lung and Blood Institute (R01-HL096624) (although the grants supported different aims, they employed the same patient population, study design, and data collection, and were combined for the purpose of these analyses); no financial relationships with any organizations that might have an interest in the submitted work in the previous three years; no other relationships or activities that could appear to have influenced the submitted work.

Ethical approval: The protocol was submitted and approved by the institutional review boards of each participating institution.

Transparency: All authors affirm that this manuscript is an honest, accurate, and transparent account of the study being reported; that no important aspects of the study have been omitted; and that any discrepancies from the study as planned have been explained.

Data sharing: No additional data available.

1 Wijeysundera HC, Nallamothu BK, Krumholz HM, Tu JV, Ko DT. Meta-analysis: effects of percutaneous coronary intervention versus medical therapy on angina relief. Ann Intern Med 2010;152:370-9.

2 Boden WE, O'Rourke RA, Teo KK, Hartigan PM, Maron DJ, Kostuk WJ, et al. Optimal medical therapy with or without PCI for stable coronary disease. N Engl J Med 2007;356:1503-16.

3 Trikalinos TA, Alsheikh-Ali AA, Tatsioni A, Nallamothu BK, Kent DM. Percutaneous coronary interventions for non-acute coronary artery disease: a quantitative 20-year synopsis and a network meta-analysis. Lancet 2009;373:911-8.

4 Stergiopoulos K, Brown DL. Initial coronary stent implantation with medical therapy vs medical therapy alone for stable coronary artery disease: meta-analysis of randomized controlled trials. Arch Intern Med 2012;172:312-9.

5 Pursnani S, Korley F, Gopaul R, Kanade P, Chandra N, Shaw RE, et al. Percutaneous coronary intervention versus optimal medical therapy in stable coronary artery disease: a systematic review and meta-analysis of randomized clinical trials. Circ Cardiovasc Interv 2012;5:476-90.

6 Kuhn EM, Hartz AJ, Baras M. Correlation of rates of coronary artery bypass surgery, angioplasty, and cardiac catheterization in 305 large communities for persons age 65 and older. Health Serv Res 1995;30:425-36.

7 Chassin MR, Brook RH, Park RE, Keesey J, Fink A, Kosecoff J, et al. Variations in the use of medical and surgical services by the Medicare population. N Engl J Med 1986;314:285-90.

8 Finn SD, Gardin JM, Abrams J, Berra K, Blankenship JC, Dallas AP, et al. 2012 ACCF/AHA/ACP/AATS/PCNA/SCAI/STS guideline for the diagnosis and management of patients with stable ischemic heart disease. A report of the American College of Cardiology Foundation/American Heart Association Task Force on Practice Guidelines, and the American College of Physicians, American Association for Thoracic Surgery, Preventive Cardiovascular Nurses Association, Society for Cardiovascular Angiography and Interventions, and Society of Thoracic Surgeons. J Am Coll Cardiol 2012;60:e44-164.

9 Holmes DR Jr, Gersh BJ, Whitlow P, King SB 3rd, Dove JT. Percutaneous coronary intervention for chronic stable angina: a reassessment. JACC Cardiovasc Interv 2008; 1:34-43.

10 Beller GA. President's page: geographic variations in delivery of cardiovascular care: an issue of great importance to cardiovascular specialists. J Am Coll Cardiol 2000;36:652-5.

11 Wennberg DE, Birkmeyer JD, ed. The Dartmouth atlas of cardiovascular health care. AHA Press, 1999.

12 Rothberg MB, Sivalingam SK, Ashraf J, Visintainer P, Joelson J, Kleppel R, et al. Patients and cardiologists' perceptions of the benefits of percutaneous coronary intervention for stable coronary disease. Ann Intern Med 2010;153:307-13.

13 Kimble LP, King KB. Perceived side effects and benefits of coronary angioplasty in the early recovery period. Heart Lung 1998;27:308-14.

14 Holmboe ES, Fiellin DA, Cusanelli E, Remetz M, Krumholz HM. Perceptions of benefit and risk of patients undergoing first-time elective percutaneous coronary revascularization. $J$ Gen Intern Med 2000;15:632-7.

15 Ozkan O, Odabasi J, Ozcan U. Expected treatment benefits of percutaneous transluminal coronary angioplasty: the patient's perspective. Int J Cardiovasc Imaging 2008:24:567-75.

16 Chandrasekharan DP, Taggart DP. Informed consent for interventions in stable coronary artery disease: problems, etiologies, and solutions. Eur J Cardiothorac Surg 2011;39:912-7.

17 Lee JH, Chuu K, Spertus J, Cohen DJ, James AG, Tang F, et al. Patients overestimate the potential benefits of elective percutaneous coronary intervention. Mo Med 2012;109:79-84.

18 Kee F, McDonald P, Gaffney B. Risks and benefits of coronary angioplasty: the patients perspective: a preliminary study. Qual Health Care 1997;6:131-9.

19 Whittle J, Conigliaro J, Good CB, Kelley ME, Skanderson M. Understanding of the benefits of coronary revascularization procedures among patients who are offered such procedures. Am Heart J 2007; 154:662-8.

20 Spertus J GE, Chhatriwalla A. Testing an evidence-based, individualized informed consent form to improve patients' experiences with PCl. Circulation 2011;124:2365-74.

21 Spertus JA, Winder JA, Dewhurst TA, Deyo RA, Prodzinski J, McDonell M, et al. Development and evaluation of the Seattle angina questionnaire: a new functional status measure for coronary artery disease. J Am Coll Cardiol 1995;25:333-41.

22 Rose GA, Blackburn H. Cardiovascular survey methods. Monogr Ser World Health Organ 1968;56:1-188.

23 Jenkins $C D$, Stanton BA, Jono RT. Quantifying and predicting recovery after heart surgery. Psychosom Med 1994;56:203-12. 


\section{What is already known on this topic}

Several studies suggest that patients have a limited understanding of the role of an elective percutaneous coronary intervention (PCI) in the management of stable coronary artery disease and overestimate its benefits

However, limitations in the generalizability of these studies include small cohort sizes, single center studies, or non-contemporary data Moreover, no study has examined how patients' perceptions of elective PCI vary by site

\section{What this study adds}

Of nearly 1000 patients interviewed with stable coronary artery disease undergoing an elective $\mathrm{PCl}$ across 10 US hospitals, most had a poor understanding of the indication and benefits of $\mathrm{PCl}$

The way each site obtained informed consent varied substantially, and after adjustment for patient characteristics patients' perceptions varied significantly across sites

Important opportunities exist to improve the process, reliability, and quality of informed consent for $\mathrm{PCl}$ and hospital level interventions may be an important step in improving patient understanding

24 Cleary PD, Epstein AM, Oster G, Morrissey GS, Stason WB, Debussey S, et al. Health-related quality of life among patients undergoing percutaneous transluminal coronary angioplasty. Med Care 1991;29:939-50.

25 Austin PC, Tu JV, Alter DA. Comparing hierarchical modeling with traditional logistic regression analysis among patients hospitalized with acute myocardial infarction: should we be analyzing cardiovascular outcomes data differently? Am Heart J 2003:145:27-35.

26 Larsen K, Merlo J. Appropriate assessment of neighborhood effects on individual health: integrating random and fixed effects in multilevel logistic regression. Am J Epidemiol 2005;161:81-8.

27 Merlo J, Chaix B, Ohlsson H, Beckman A, Johnell K, Hjerpe P, et al. A brief conceptual tutorial of multilevel analysis in social epidemiology: using measures of clustering in multilevel logistic regression to investigate contextual phenomena. J Epidemiol Community Health 2006:60:290-7.

28 Arnold SV, Decker C, Ahmad H, Olabiyi O, Mundluru S, Reid KJ, et al. Converting the informed consent from a perfunctory process to an evidence-based foundation for patient decision making. Circ Cardiovasc Qual Outcomes 2008;1:21-8.

29 Krumholz HM, Barreto-Filho J, Jones PG, Li Y, Spertus JA. Decision-making preferences among patients with an acute myocardial infarction. JAMA Intern Med 2013;173:1252-7.

30 Deber RB, Kraetschmer N, Irvine J. What role do patients wish to play in treatment decision making? Arch Intern Med 1996;156:1414-20.

31 Moulton B, King JS. Aligning ethics with medical decision-making: the quest for informed patient choice. J Law, Med Ethics 2010;38:85-97.

32 Lin GA, Dudley RA, Redberg RF. Cardiologists' use of percutaneous coronary interventions for stable coronary artery disease. Arch Intern Med 2007;167:1604-9.

33 Bakalar N. No extra benefits are seen in stents for coronary artery disease: New York Times; 2012. www.nytimes.com/2012/02/28/health/stents-show-no-extra-benefits-forcoronary-artery-disease.html? pagewanted=all\&_r $=0$.

34 Feder BJ. First, a new artery stent study; now, questions about what it all means. New York Times; 2007. www.nytimes.com/2007/03/28/business/28place.html?_r=1.

35 Winstein KJ. A simple health-care fix fizzles out. Wall Street Journal; 2010. http://online. ws..com/article/SB10001424052748703652104574652401818092212.html.

36 Wennberg JE. Practice variations and health care reform: connecting the dots. Health Aft (Millwood) 2004;Suppl Variation:Var140-4.

37 Wennberg DE, Wennberg JE. Addressing variations: is there hope for the future? Health Aff (Millwood) 2003;Suppl Web Exclusives:W3-614-7.

38 Head SJ, Kaul S, Mack MJ, Serruys PW, Taggart DP, Holmes DR Jr, et al. The rationale for Heart Team decision-making for patients with stable, complex coronary artery disease. Eur Heart J 2013;34:2510-8.
39 Coylewright M, Shepel K, Leblanc A, Pencille L, Hess E, Shah N, et al. Shared decision making in patients with stable coronary artery disease: $\mathrm{PCl}$ choice. PloS One 2012;7:e49827.

40 Tait AR, Voepel-Lewis T, Moscucci M, Brennan-Martinez CM, Levine R. Patient comprehension of an interactive, computer-based information program for cardiac catheterization: a comparison with standard information. Arch Intern Med 2009:169:1907-14.

41 Morgan MW, Deber RB, Llewellyn-Thomas HA, Gladstone P, Cusimano RJ, O'Rourke $\mathrm{K}$, et al. Randomized, controlled trial of an interactive videodisc decision aid for patients with ischemic heart disease. J Gen Intern Med 2000;15:685-93.

42 Liao L, Jollis J, DeLong E, Peterson E, Morris K, Mark D. Impact of an interactive video on decision making of patients with ischemic heart disease. J Gen Intern Med 1996;11:373-6.

43 Gershon RR, Stone PW, Bakken S, Larson E. Measurement of organizational culture and climate in healthcare. J Nurs Adm 2004;34:33-40.

44 Glickman SW, Baggett KA, Krubert CG, Peterson ED, Schulman KA. Promoting quality: the health-care organization from a management perspective. Int $J$ Qual Health Care 2007;19:341-8.

45 Pearson P, Steven A, Howe A, Sheikh A, Ashcroft D, Smith P. Learning about patient safety: organizational context and culture in the education of health care professionals. $\checkmark$ Health Serv Res Policy 2010;15(suppl 1):4-10.

46 Scott T, Mannion R, Marshall M, Davies H. Does organisational culture influence health care performance? A review of the evidence. J Health Serv Res Policy 2003;8:105-17.

47 Davies HT, Nutley SM, Mannion R. Organisational culture and quality of health care. Qual Health Care 2000;9:111-9.

48 Bellot J. Defining and assessing organizational culture. Nurs Forum 2011;46:29-37.

\section{Accepted: 19 August 2014}

\section{Cite this as: BMJ 2014;349:95309}

This is an Open Access article distributed in accordance with the Creative Commons Attribution Non Commercial (CC BY-NC 3.0) license, which permits others to distribute, remix, adapt, build upon this work non-commercially, and license their derivative works on different terms, provided the original work is properly cited and the use is non-commercial. See: http://creativecommons.org/licenses/by-nc/3.0/. 


\section{Tables}

Table 1 | Volume of patients with stable coronary artery disease seen by interventional cardiologists (operators)

No of patients No $(\%)$ of operators $(n=135)$

\begin{tabular}{ll}
1 to $<5$ & $63(47)$ \\
\hline 5 to $<10$ & $39(29)$ \\
\hline 10 to $<15$ & $18(13)$ \\
\hline 15 to $<20$ & $4(3)$ \\
\hline 20 to $<25$ & $6(4)$ \\
\hline 25 to 46 & $5(4)$ \\
\hline
\end{tabular}


Table 2| Qualitative description of the informed consent process at each site

Professional responsible for signed consent

\begin{tabular}{ccc}
\cline { 2 - 2 } Site & Operator & N \\
\hline 2 & +++ \\
\hline 3 & +++ \\
\hline 4 & + \\
\hline 5 & ++ \\
\hline 6 & ++ \\
\hline 7 & +++ \\
\hline 8 & + \\
\hline 9 & ++ \\
\hline 10 & +++ \\
\hline
\end{tabular}

$\mathrm{ND}=$ not done.

+++=predominant mode; ++=occasional mode; +=rare mode

$+++\quad \mathrm{ND}$

ND $\quad+++$

ND $\quad++$

$+++N D$

$++\quad \mathrm{ND}$

ND $\quad++$

ND $\quad+++$

Setting for signed consent

Office Holding room for outpatient Room for inpatient provided to patient

ND

$++\quad$ ND

+ ND $\quad+++\quad+++$

$\begin{array}{llll}++ & \text { ND } & +++ & ++\end{array}$

ND $\quad+++$

$+++$

ND + +++ ++

ND ND $\quad+++\quad++$

+ ND $\quad++\quad++$

ND ND $\quad+++\quad+++$

ND ND

$\begin{array}{lll}++ & \text { ND } & \text { ND } \\ + & \text { ND } & +++\end{array}$

$++$ 
Table 3| Characteristics of patients with stable coronary artery disease who completed the interview (n=991)

\begin{tabular}{|c|c|}
\hline Characteristics & No $(\%)^{*}$ \\
\hline Mean (SD) age (years) & $65.0(10.6)$ \\
\hline \multicolumn{2}{|l|}{ Sex: } \\
\hline Male & $745(75.2)$ \\
\hline Female & $246(24.8)$ \\
\hline \multicolumn{2}{|l|}{ Race: } \\
\hline White & $906(92.9)$ \\
\hline Black/African-American & $37(3.8)$ \\
\hline Other & $32(3.3)$ \\
\hline Married & $737(74.7)$ \\
\hline Less than high school education & $74(7.5)$ \\
\hline No insurance & $6(0.6)$ \\
\hline \multicolumn{2}{|l|}{ Insurance type: } \\
\hline Private & $780(83.7)$ \\
\hline Military & $13(1.4)$ \\
\hline Medicare & $453(48.6)$ \\
\hline Indian health service & $2(0.2)$ \\
\hline Medicaid & $29(3.1)$ \\
\hline State specific & $7(0.8)$ \\
\hline Avoided care owing to cost & $86(8.7)$ \\
\hline Prior myocardial infarction & $250(25.2)$ \\
\hline Prior percutaneous coronary intervention & $432(43.6)$ \\
\hline Prior coronary artery bypass grafting & $195(19.7)$ \\
\hline Stress imaging performed & $622(62.8)$ \\
\hline Mean (SD) Seattle angina questionnaire physical Limitation Score and (SD) & $77.9(22.8)$ \\
\hline \multicolumn{2}{|l|}{$S A Q$ angina frequency score: } \\
\hline 0-30 (daily) & $78(7.9)$ \\
\hline $31-60$ (weekly) & $284(28.7)$ \\
\hline 61-99 (monthly) & $310(31.3)$ \\
\hline 100 (none) & $319(32.2)$ \\
\hline \multicolumn{2}{|l|}{ SAQ quality of life score: } \\
\hline $0-24$ (poor) & $69(7.0)$ \\
\hline 25-49 (fair) & $266(26.9)$ \\
\hline $50-74$ (good) & $304(30.7)$ \\
\hline 75-100 (excellent) & $350(35.4)$ \\
\hline \multicolumn{2}{|l|}{ Rose dyspnea score: } \\
\hline 0 & $304(31.1)$ \\
\hline 1 & $177(18.1)$ \\
\hline 2 & $167(17.1)$ \\
\hline 3 & $175(17.9)$ \\
\hline 4 & 155 (15.8) \\
\hline
\end{tabular}

$S A Q=S e a t t l e$ angina questionnaire.

*Unless stated otherwise. 
Table 4 | Characteristics of operators who performed the percutaneous coronary intervention procedure $(n=135)$

\begin{tabular}{lc} 
Characteristics & No (\%) \\
Mean (SD) age (years) & $50.7(8.4)$ \\
\hline Sex: & \\
\hline Female & $127(9.9)$ \\
\hline Male & $32(23.7)$ \\
\hline Medical school: & $103(76.3)$ \\
\hline Foreign & \\
\hline USA/Canada & $17.6(9.3)$ \\
\hline Years in practice: & $16.0(10.0-25.0)$ \\
\hline Mean (SD) & $130(96.3)$ \\
\hline Median (interquartile range) & $111(82.2)$ \\
\hline Board certification in cardiology & \\
\hline Board certification in interventional cardiology & $27.2(79.4)$ \\
\hline No of publications in journals & $6.0(0.0-24.0)$ \\
\hline Mean (SD) & \\
\hline Median (interquartile range) & $37(27.4)$ \\
\hline No of publications: & $50(37.0)$ \\
\hline 0 & $27(20.0)$ \\
\hline $1-9$ & $21(15.6)$ \\
\hline $10-49$ & \\
\hline$\geq 50$ & \\
\hline &
\end{tabular}

*Unless stated otherwise. 
Table 5| Reported perceptions of percutaneous coronary intervention by patients with stable coronary artery disease

Perceived benefit Study average (\%) Hospital range (\%)

Extend life

$90 \quad 80-97$

Prevent myocardial infarction

79-97

Save life

88

Decrease symptoms

69

Improve abnormality

$67 \quad 52-87$

Other

52

32-68

Emergent procedure

$20 \quad 4-38$


Table 6 | Frequency of benefits of percutaneous coronary intervention chosen by patients ( $n=991)$

No of benefits selected Frequency (\%)

\begin{tabular}{lc}
0 & 0.4 \\
\hline 1 & 5.3 \\
\hline 2 & 8.7 \\
\hline 3 & 17.8 \\
\hline 4 & 29.6 \\
\hline 5 & 29.3 \\
\hline 6 & 8.6 \\
\hline 7 & 0.4 \\
\hline
\end{tabular}




\section{Figures}

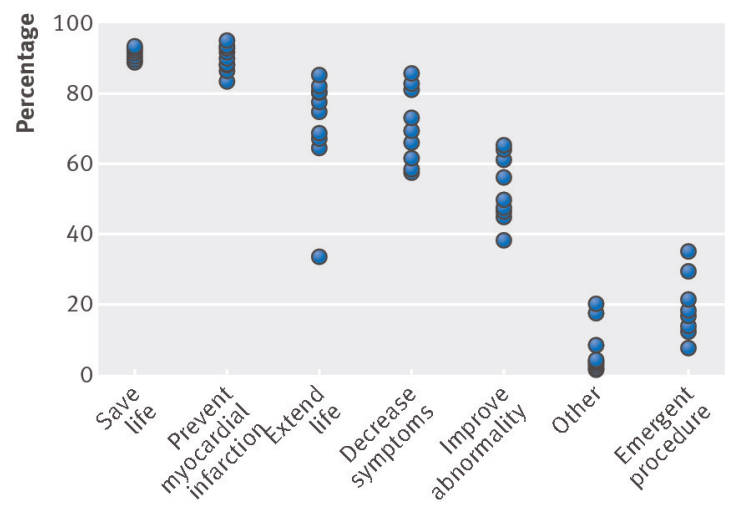

Fig 1 Proportion of patients with stable coronary artery disease with various perceptions of their elective percutaneous coronary intervention (PCI) by site $(n=10)$

\begin{tabular}{|c|c|c|}
\hline Perception of PCI & $\begin{array}{l}\text { Median odds ratio* } \\
\qquad(95 \% \mathrm{Cl})\end{array}$ & $\begin{array}{c}\text { Median odds ratio* } \\
(95 \% \mathrm{Cl})\end{array}$ \\
\hline \multicolumn{3}{|l|}{ Extend life } \\
\hline Operator & $\longrightarrow$ & $1.46(1.00$ to 2.11$)$ \\
\hline Hospital & $\rightarrow$ & $1.38(1.00$ to 2.24$) \dagger$ \\
\hline \multicolumn{3}{|c|}{ Prevent myocardial infarction } \\
\hline Operator & $\rightarrow-$ & 1.31 (1.00 to 1.91$)$ \\
\hline Hospital & $\rightarrow$ & 1.72 (1.29 to 2.94$)$ \\
\hline \multicolumn{3}{|l|}{ Save life } \\
\hline Operator & $=$ & $1.06(1.00$ to 1.57$)$ \\
\hline Hospital & - & 2.04 (1.57 to 3.54$)$ \\
\hline \multicolumn{3}{|c|}{ Decrease symptoms } \\
\hline Operator & 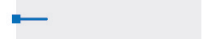 & $1.00(1.00$ to 1.40$)$ \\
\hline Hospital & $\rightarrow$ & 1.74 (1.36 to 2.84$)$ \\
\hline \multicolumn{3}{|c|}{ Improve abnormality } \\
\hline Operator & 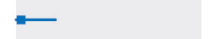 & $1.06(1.00$ to 1.53$)$ \\
\hline Hospital & $\rightarrow$ & 1.52 (1.23 to 2.23$)$ \\
\hline \multicolumn{3}{|l|}{ Other } \\
\hline Operator & $\longrightarrow$ & 1.58 (1.00 to 2.45$)$ \\
\hline Hospital & $\longrightarrow$ & 3.11 (1.83 to 9.17$)$ \\
\hline \multicolumn{3}{|c|}{ Emergent procedure } \\
\hline Operator & $\longrightarrow$ & 1.34 (1.00 to 1.83$)$ \\
\hline \multirow[t]{2}{*}{ Hospital } & $\longrightarrow$ & 1.95 (1.44 to 3.52$)$ \\
\hline & 1 & \\
\hline
\end{tabular}

Fig 2 Median odds ratios (95\% confidence intervals) of patients' perceptions of the urgency and benefits of elective percutaneous coronary intervention by hospitals and operators within a hospital. *Adjusted for patient and operator (interventional cardiologist) characteristics. †Confidence interval comes close to 1.00 but does not include it, $\mathrm{P} \leq 0.05$ 